\title{
Preparation of cross-linked cellulose nanofibril aerogel with water absorbency and shape recovery
}

\author{
Chae Hoon Kim • Hye Jung Youn · Hak Lae Lee
}

Received: 8 April 2015/Accepted: 31 August 2015/Published online: 8 September 2015

(C) The Author(s) 2015. This article is published with open access at Springerlink.com

\begin{abstract}
Cellulose nanofibril (CNF) aerogels are promising materials for various applications because of their highly porous and ultralight characteristics. The fiber network of CNF aerogels held together by hydrogen bonding and mechanical entanglement of adjacent fibers is easily destroyed when it is exposed to water. In this study, cross-linked CNF aerogels were prepared using maleic acid and sodium hypophosphite as cross-linking agents. In the first step of treatment, CNF dispersed in water was reacted with maleic acid to form an ester linkage. Sodium hypophosphite was then added to the maleic acid-functionalized CNF suspension and the suspension was rapidly frozen using liquid nitrogen. CNF aerogel was obtained after freeze drying. The cross-linking of the cellulose was formed by the reaction between the carbon-carbon double bond of maleic acid-functionalized CNF and hypophosphite. The cross-linked CNF aerogel exhibited good network stability in water and springiness after compression.
\end{abstract}

C. H. Kim · H. J. Youn · H. L. Lee $(\bowtie)$

Department of Forest Sciences, Research Institute of Agriculture and Life Sciences, College of Agriculture and Life Sciences, Seoul National University, 1 Gwanak-ro, Gwanak-gu, Seoul 151-921, Republic of Korea e-mail: lhakl@snu.ac.kr

C. H. Kim e-mail: chkim5220@snu.ac.kr

H. J. Youn

e-mail: page94@snu.ac.kr
Keywords Cellulose nanofibril · Aerogel · Crosslinking $\cdot$ Water absorbency $\cdot$ Shape recovery

\section{Introduction}

Cellulose nanofibril (CNF) is a nano-scale fibrous material which can be obtained from the cellulose fiber using various types of mechanical shearing treatments. The preparation of CNF was reported in early 80 s by Turbak et al. (1983) and Herrick et al. (1983). It has been shown that cellulose fibrils with nanometer width can be produced by the high pressure shearing action during the homogenizing process. Besides the high pressure homogeziner (Nakagaito and Yano 2004; Iwamoto et al. 2005), grinder (Iwamoto et al. 2007; Sim et al. 2015), microfluidizer (Zimmermann et al. 2010; Siqueira et al. 2010), cryo-crushing equipment (Dufresne et al. 1997; Alemdar and Sain 2007), refiner (Heiskanen et al. 2012), and high speed blender (Uetani and Yano 2011) have been employed to produce CNF. Producing CNF by means of mechanical disintegration of cellulose fiber inevitably requires a great deal of energy. To reduce the energy consumption several pretreatment techniques including oxidation (Saito et al. 2006, 2007; Isogai et al. 2011), carboxymethylaion (Aulin et al. 2010; Taipale et al. 2010), and enzymatic hydrolysis (Pääkkö et al. 2007; Henriksson et al. 2007; Siqueira et al. 2010) of cellulose fiber have been suggested. 
The morphological characteristics of CNF may vary depending upon the type of mechanical treatment, degree of applied shear forces and pre-treatment. In general, CNF is an aggregation of 10-50 cellulose elementary fibrils with a complex web-like network structure. Its diameter and length range 5-50 $\mathrm{nm}$ and several $\mu \mathrm{m}$, respectively (Missoum et al. 2013).

CNF has a range of possible applications because of its high specific surface area and strong mechanical properties. For example, CNF can be used as a reinforcing agent for biocomposite materials (Shibata and Nakai 2010) or building material for translucent sheets (Yano and Nakahara 2004). Another promising application of $\mathrm{CNF}$ is to prepare cellulose aerogel (Pääkkö et al. 2008).

Aerogels are highly porous and ultra-lightweight materials formed by removing the liquid dispersion medium from the gel without collapsing the network structure (Hüsing and Schubert 1998). Since aerogels were first introduced by Kistler (1931), most aerogels have been prepared by the sol-gel process or by the supercritical fluid drying of inorganic metal-oxide materials or polymers (Pierre and Pajonk 2002). These processes, however, require multiple solvent-exchange steps and use of the considerable amount of energy, which brings both environmental and economic issues that should be resolved for their wider acceptance.

Because of the morphological characteristics of $\mathrm{CNF}$, they entangle mechanically in aqueous suspension and form a homogenous gel structure at a very low concentration (Missoum et al. 2013). The capability of CNF to form interfiber hydrogen bonds allows the preparation of aerogel by removing the water. In particular, rapid freezing and freeze-drying of the CNF hydrogel makes the preparation of the highly porous and ultra-lightweight CNF aerogel possible. Unlike conventional aerogels, there is no need for a crosslinking treatment for the CNF aerogel. Solvent exchange or supercritical drying processes are also unnecessary in preparing CNF hydrogel (Cervin et al. 2012; Chen et al. 2011; Aulin et al. 2010).

Mechanical flexibility of CNF aerogels and the possibility of adapting various functionalities by utilizing the abundant hydroxyl groups for chemical modifications allow a number of potential applications of the CNF aerogels. Potential applications of CNF aerogels include kinetic energy absorbers, thermal and acoustic insulating materials, reinforcing platforms, drug carrier, tissue engineering scaffolds and others (Sehaqui et al. 2010).

CNF aerogels, however, show poor structural stability in aqueous condition. This is because the network structure of the $\mathrm{CNF}$ aerogel relies on the interfiber hydrogen bonding and mechanical entanglement that is partly destroyed in aqueous condition. Structural stability of CNF aerogels in aqueous condition can be improved by covalent cross-linking. Zhang et al. (2012) have reported cross-linked CNF aerogels which showed significantly improved network stability in wet state using polyamide-epichlorohydrin resin. Syverud et al. (2015) have shown that the stability of CNF hydrogel can be improved using aldehyde groups as a cross-linking agent. It is worthwhile, however, to find a new approach to improve the stability of the CNF aerogel in aqueous conditions to have more possibilities to control the properties of aerogels.

In this study, a covalently cross-linked CNF aerogel was prepared using maleic acid and sodium hypophosphite in aqueous media. Maleic acid was grafted onto cellulose fiber, and covalent cross-linking of CNF was formed by the reaction between maleic acid and hypophosphite. The network stability of the cross-linked $\mathrm{CNF}$ aerogel in a wet state as well as their physical and structural characteristics were investigated.

\section{Experimental}

\section{Preparation of CNF}

CNF was prepared using a grinding method according to Sim et al. (2015). Once-dried, commercial bleached eucalyptus kraft pulp was used as a raw material for the preparation of the CNF. Dried pulp sheets were dispersed in deionized water and refined using a laboratory Valley beater to a freeness value of $450 \mathrm{~mL}$ CSF. The consistency of the beaten pulp suspension was adjusted to $2 \%$, after which the suspension was subjected to a mechanical treatment using a grinder (Super Masscollider MKCA6-3, Masuko Sanguo Co.). The rotation speed and gap of the grinding stone were $1500 \mathrm{rpm}$ and $-60 \mu \mathrm{m}$, respectively. After 40 cycles of the grinding treatments, CNF hydrogel was obtained. Scanning electron microscope (SEM) images of the prepared CNF were shown in Fig. 1. 
Fig. 1 SEM images of prepared $\mathrm{CNF}$
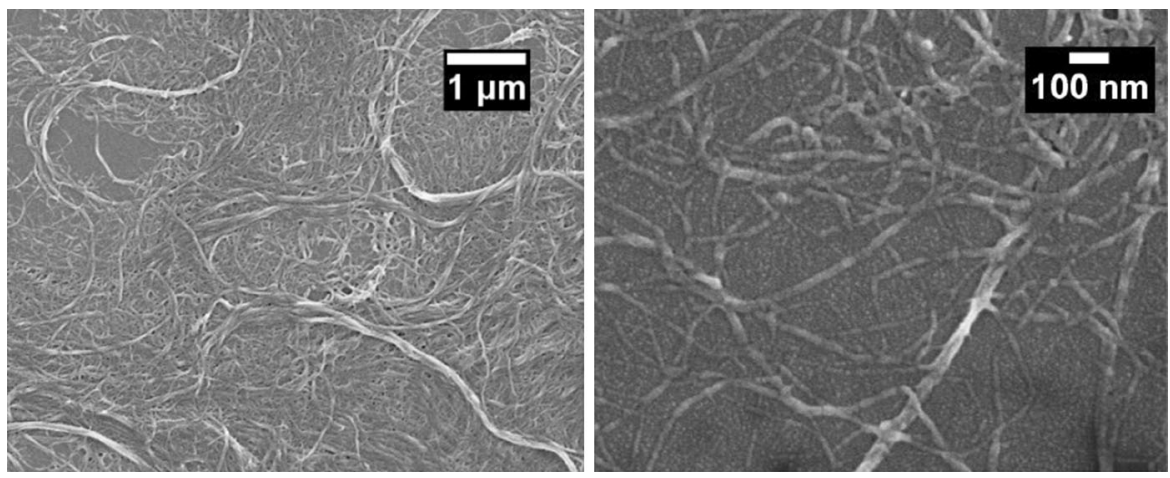

The chemical composition of the CNF was determined by the NREL (National Renewable Energy Laboratory, USA) method (NREL/TP-510-42618). The contents of $\alpha$-cellulose, hemicellulose, lignin and ash were found to be $79.4,18.7,1.5$, and $0.5 \%$, respectively. To confirm esterification of cellulose with maleic acid, filter paper CNF was also prepared from pure cellulose. Advantac 5C grade filter paper and the same procedure described above was used in preparing filter paper based CNF for FT-IR spectroscopy.

\section{Preparation of maleic acid-functionalized CNF}

For the cross-linking reaction of the CNF aerogel, analytical-grade maleic acid and sodium hypophosphite, supplied by Sigma-Aldrich, were used. Maleic acid and sodium hypophosphite were added to the CNF suspension. The dosage of maleic acid based on the weight of anhydroglucose unit (AGU) of cellulose was 10 . To determine this dosage ratio, it was assumed that CNF was composed of cellulose only. Sodium hypophosphite was added as a catalyst for the esterification reaction of cellulose and maleic acid. The dosage of sodium hypophosphite was $5 \mathrm{wt} \%$ of the maleic acid. After mixing for $30 \mathrm{~min}$, the CNF suspension containing maleic acid and sodium hypophosphite was heated to $120{ }^{\circ} \mathrm{C}$ and kept at that temperature for $30 \mathrm{~min}$ in an autoclave for esterification. Unreacted chemicals after the esterification reaction were removed by centrifugal washing at $3000 \mathrm{~g}$ for $10 \mathrm{~min}$. After which the maleic acidfunctionalized CNF (CMF-MA) was settled down and the clear supernatant containing unreacted maleic acid and sodium hypophosphite was removed. The CNFMA pad was re-dispersed in deionized water and successive washing steps were carried out. After six times of centrifugal washings, CNF-MA was collected and used for cross-linking reaction. Maleic acidfunctionalized filter paper CNF (filter paper CNF-MA) was also prepared with same procedure to verify the esterification of cellulose using FT-IR spectroscopy analysis.

Preparation of the cross-linked CNF aerogel

Twenty wt $\%$ of sodium hypophosphite based on the oven dried weight of CNF-MA was added to the CNFMA suspension. The CNF-MA hydrogel mixed with sodium hypophosphite of which solids content was adjusted to $1.0-3.0 \mathrm{wt} \%$ was poured into polystyrene cuvette molds. The CNF hydrogels in the cuvette were precooled by placing in a refrigerator at $4{ }^{\circ} \mathrm{C}$ for overnight to minimize the fracture of the frozen $\mathrm{CNF}$ suspension during the subsequent rapid freezing step (Zheng et al. 2014). The precooled CNF hydrogels were rapidly frozen at $-196{ }^{\circ} \mathrm{C}$ using liquid nitrogen. These frozen samples were subjected to freeze-drying at $-80{ }^{\circ} \mathrm{C}$ and $5 \mathrm{mmHg}$ using a freeze-dryer (FD8518, Ilshin Lab) for 3 days, and CNF aerogels were obtained. For cross-linking of CNF, the CNF aerogels were placed in a convection oven and cured for $10 \mathrm{~min}$ at $170{ }^{\circ} \mathrm{C}$. Cross-linked $\mathrm{CNF}$ aerogels were cut into cubic shapes with a dimension of $10 \times 10 \times 10 \mathrm{~mm}^{3}$ and conditioned in a control room at $23{ }^{\circ} \mathrm{C}$ and $50 \% \mathrm{RH}$.

\section{Characterization of cross-linked CNF aerogel}

Maleic acid-functionalized filter paper CNF (filter paper CNF-MA) was used for FT-IR spectroscopy to identify the ester linkage between the maleic acid and cellulose. After the esterification process, the filter 
paper CNF-MA suspensions were treated with a $0.1 \mathrm{M}$ $\mathrm{NaOH}$ solution at room temperature for $1 \mathrm{~h}$ to convert carboxylic acid to carboxylate anion. Then it was vacuum-filtered to obtain a filter paper CNF-MA film and dried in a convection oven for 2 days at $40{ }^{\circ} \mathrm{C}$. FT-IR spectra of the filter paper CNF-MA film were obtained using a FT-IR spectrometer (Cary 660, Agilent) in ATR mode. For each spectrum, 32 scans were collected with a range of $600-4000 \mathrm{~cm}^{-1}$ at a resolution of $4 \mathrm{~cm}^{-1}$.

A scanning electron microscope (SEM, Auriga, Carl Zeiss) was used for the observation of the crosslinked CNF aerogels. Cross-linked CNF aerogel samples were frozen at $-196{ }^{\circ} \mathrm{C}$ using liquid nitrogen, and its cross-section was prepared by fracturing the frozen sample. Then sample was attached to a sample stub with a double-sided adhesive conductive carbon tape and sputter-coated with platinum.

The specific surface area of the aerogel was determined using nitrogen adsorption/desorption measurements (ASAP 2020, Micromeritics Instruments Co.) at $-196{ }^{\circ} \mathrm{C}$. Before the adsorption measurement, the samples, dried at $105{ }^{\circ} \mathrm{C}$ for $24 \mathrm{~h}$ to remove moisture, were degassed at $120{ }^{\circ} \mathrm{C}$ for $4 \mathrm{~h}$ for the complete elimination of moisture. Both the adsorption and desorption isotherms were measured, and the surface area was determined from the adsorption curve by the Brunauer-Emmett-Teller (BET) method.

To measure the moisture uptake of the cross-linked CNF aerogel, saturated lithium chloride, calcium chloride, and sodium chloride solutions were used to control the relative humidity of air to 15,37 , and $75 \%$ $\mathrm{RH}$ at $23{ }^{\circ} \mathrm{C}$, respectively. These saturated salt solutions were placed in a humidity chamber and the weight changes of the fully dried cross-linked aerogels were measured to determine the moisture contents.

To evaluate the compressive strength and shaperecovery performance of the cross-linked CNF aerogel, a texture analyzer (TA XT Plus, Stable Micro Systems, Ltd.) with a $50 \mathrm{~mm}$ cylindrical probe and a $50 \mathrm{~kg}$ load cell was used. During the compression of cross-linked CNF aerogel, no fracture point or inflection point was observed on the stress-strain curve until $90 \%$ of its original dimension. The compressive stress at a strain level of $90 \%$ was determined as the compress strength. In this test, the probe speed was adjusted to $1 \mathrm{~mm} / \mathrm{sec}$.

To evaluate the shape-recovery performance of the cross-linked CNF aerogel in the wet state, a texture

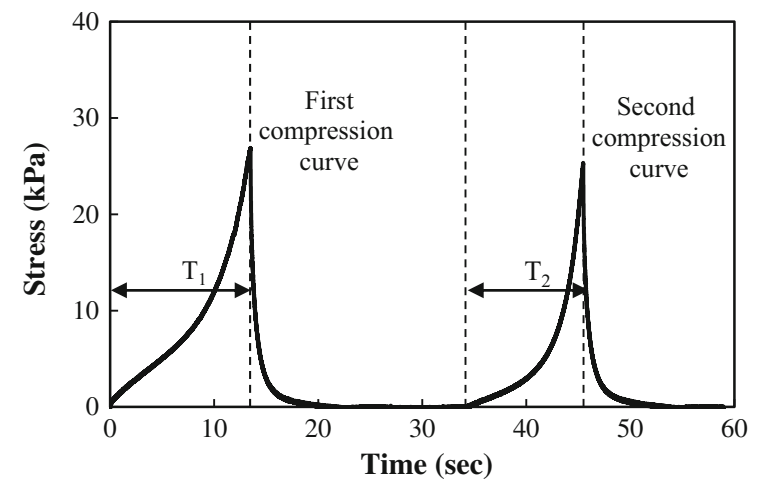

Fig. 2 Time-stress curve in TPA test

profile analysis (TPA) was carried out. This test consists of two successive $70 \%$ strain compressions of the cross-linked CNF aerogel, as shown in Fig. 2. Springiness, the recovery ratio of the deformed material after the removal of a deforming force, was obtained as in Eq. (1). $T_{1}$ and $T_{2}$ represent the time required for $70 \%$ compression of the cross-linked CNF aerogel in the first and second compression cycles, respectively. When the springiness value is closer to one, the cross-linked CNF aerogel easily recovers to its original shape after compression. For the TPA test, the probe speed was adjusted to $0.5 \mathrm{~mm} /$ s. The time interval between the first and second compression was $5 \mathrm{~s}$.

Springiness $=\frac{T_{2}}{T_{1}}$

\section{Results and discussion}

\section{Esterification of maleic acid onto $\mathrm{CNF}$}

The cross-linking of CNF takes place in two separate reactions when maleic acid and hypophosphite were used. The first step is an esterification reaction between maleic acid and hydroxyl group of cellulose. In an aqueous solution, maleic acid forms a fivemember cyclic anhydride by the dehydration of two carboxylic groups. This intermediate reacts with the hydroxyl group of cellulose to form an ester linkage (Fig. 3). In this esterification process, sodium hypophosphite was used as a catalyst. When the reaction temperature is below $140{ }^{\circ} \mathrm{C}$, the reaction rate 


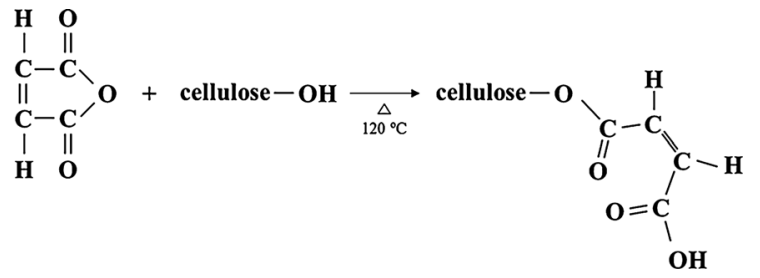

Fig. 3 Esterification between cellulose and maleic acid anhydride

of esterification is drastically increased by the presence of sodium hypophosphite (Yang et al. 2010).

The FT-IR spectra of the filter paper CNF-MA are shown in Fig. 4. As more maleic acid was added to the filter paper CNF suspension, the peak at $1570 \mathrm{~cm}^{-1}$, assigned to the $\mathrm{C}-\mathrm{O}$ stretching vibration of the ester linkage, increased. When the CNF made of hardwood kraft pulp was used, the FT-IR spectra of the CNF-MA did not show any significant difference compared to that of untreated CNF. This is because the bleached hardwood kraft pulp contains nearly $20 \%$ of hemicellulose and lignin, which gave absorption peaks at $1570 \mathrm{~cm}^{-1}$.

\section{Cross-linking of cellulose}

Polycarboxylic acids such as 1,2,3,4,-butanetetracarboxylic acid (BTCA) are used as cross-linking agents for the durable press finishes of cotton fabric because they form multiple ester linkages with cellulose (Yang et al. 2010; Yao et al. 2013). Maleic acid does not crosslink cellulose. The presence of hypophosphite ion, however, allows the cross-linking of maleic acid-

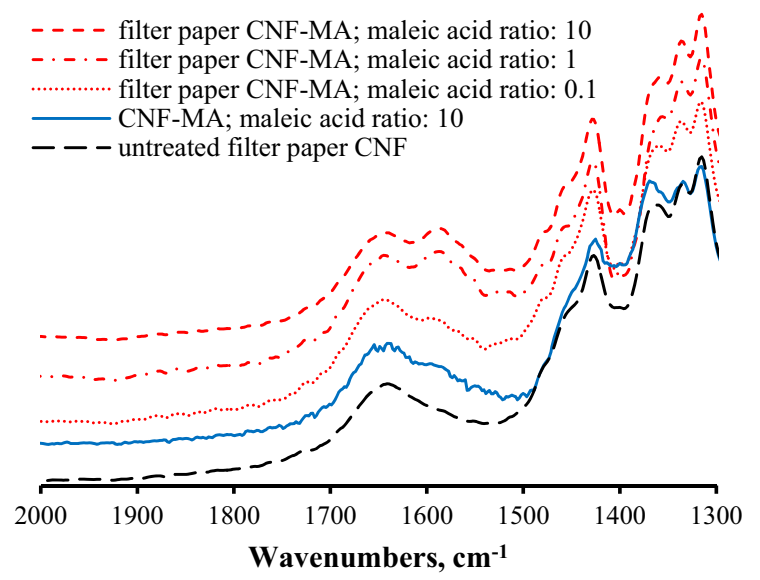

Fig. 4 FT-IR spectra of CNF-MA and filter paper CNF-MA functionalized CNF. When the reaction temperature was $120^{\circ} \mathrm{C}$ as in esterification process, sodium hypophosphite acts as a catalyst. Whereas hypophosphite ions react with carbon-carbon double bonds of maleic acid-functionalized CNF as shown in Fig. 5 (Yang et al. 2010), when the reaction temperature is higher than $140{ }^{\circ} \mathrm{C}$.

\section{Cross-linking of CNF aerogel}

In the case of typical CNF aerogels, the network structure is built by hydrogen bonds and physical entanglements between adjacent CNFs. When the typical CNF aerogels are exposed to moisture, interfiber hydrogen bonds are replaced with cellulosewater hydrogen bonds. As a result, the entire network structure of aerogels loses its strength.

The cross-linked and untreated CNF aerogels in cubic shapes $\left(10 \times 10 \times 10 \mathrm{~mm}^{3}\right)$ were immersed in deionized water and stirred for $1 \mathrm{~min}$ with a magnetic stirrer at $300 \mathrm{rpm}$. The density of aerogel was $31.5 \mathrm{~kg}$ / $\mathrm{m}^{3}$. As shown in Fig. 6, the CNF aerogel which had not undergone a cross-linking treatment disintegrated and dispersed in the water, while the cross-linked CNF aerogel maintained its original structure. This shows that the covalent cross-linking of CNF was formed through the treatment with maleic acid and hypophosphite.

In Table 1, the compressive strengths of the crosslinked and untreated CNF aerogel with density of $31.5 \mathrm{~kg} / \mathrm{m}^{3}$ were compared. The cross-linked CNF aerogel exhibited greater compressive strength in both dry and wet state; this indicates that the covalent crosslinking of CNF was formed via the reaction between the cellulose-grafted maleic acid and hypophosphite. The compressive strength of the untreated aerogels, however, was around $70 \%$ of that of the cross-linked

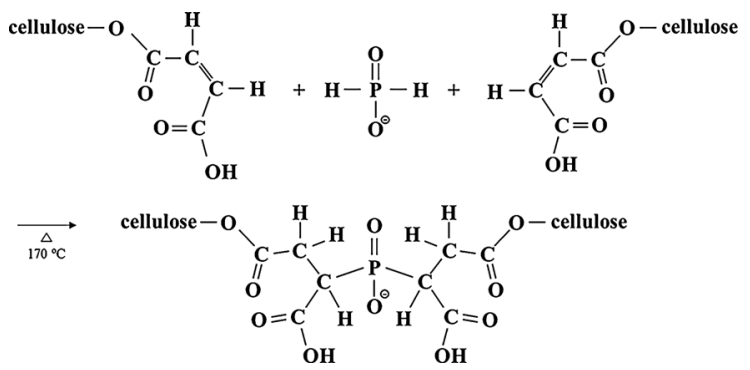

Fig. 5 Cross-linking of cellulose by the reaction between maleic acid and hypophosphite 

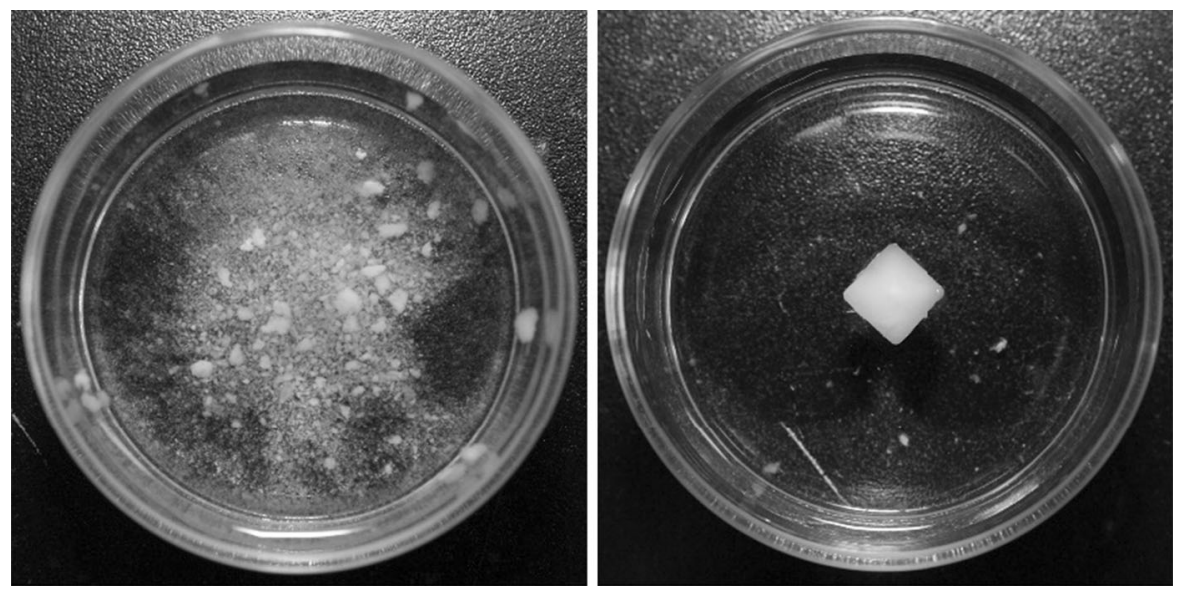

Fig. 6 Pictures of untreated CNF aerogel (left) and cross-lined CNF aerogel (right) after magnetic stirring for 1 min in water

Table 1 Compressive strength of cross-liked and untreated $\mathrm{CNF}$ aerogel in dry and wet state

\begin{tabular}{lll}
\hline & \multicolumn{2}{l}{ Compressive strength (kPa) } \\
\cline { 2 - 3 } & Dry state & Wet state \\
\hline Cross-linked CNF aerogel & $411(44)^{\text {S.D. }}$ & $180(16)$ \\
Untreated CNF aerogel & $317(35)$ & $120(13)$ \\
\hline
\end{tabular}

S.D. standard deviation

samples, which indicates that significant parts of the CNF networks are still formed via hydrogen bonding.

Internal structure of the cross-linked CNF aerogel

The water present in the CNF hydrogel was replaced with air by rapid freezing and freeze-drying, which gave CNF aerogels without a collapse or shrink (Aulin et al. 2010). In other words, the apparent volume of the CNF aerogels is equal to the original volume of the CNF suspension in the mold. Therefore, the density of aerogel is proportional to the solids content of CNF suspension (Table 2). The specific surface area of the CNF aerogel with a density of $26.7 \mathrm{~kg} / \mathrm{m}^{3}$ was $19.5 \mathrm{~m}^{2} / \mathrm{g}$, which was quite lower than that of organic or inorganic aerogels. For instance, Cai et al. (2012) reported that the specific surface area of aerogels prepared with sol-gel processes were $400-654 \mathrm{~m}^{2} / \mathrm{g}$. Our result, however, is in good agreement with previously reported values for freeze-dried CNF aerogels. Sehaqui et al. (2010) have shown that specific surface areas of $15-45 \mathrm{~m}^{2} / \mathrm{g}$ could be obtained for $\mathrm{CNF}$ aerogels with densities ranging from 7 to $103 \mathrm{~kg} / \mathrm{m}^{3}$.

Cross-sectional images of a CNF aerogel with a density of $26.7 \mathrm{~kg} / \mathrm{m}^{3}$ are shown in Fig. 7. This CNF aerogel exhibits a highly porous internal structure with open pores. It was noted that the network structure of the CNF aerogel was built with very thin nanopapers. Svagan et al. (2008) and Chen et al. (2011) have shown that randomly distributed nanofibers are forced out by the growing ice crystals and develop film-like cellulose nanopaperlets during the freeze-drying process. This nanopaper structure is commonly found in cellulose aerogels prepared by the freeze-drying of a CNF suspension (Cervin et al. 2012; Aulin et al. 2010; Sehaqui et al. 2010; Syverud et al. 2011).

Water absorption performance of the cross-linked CNF aerogel

A water-absorption test was carried out by immersing the CNF aerogels into deionized water. The CNF aerogels absorbed water instantly by wetting and

Table 2 Density of CNF aerogel and specific surface area

\begin{tabular}{lrrrr}
\hline Solids content of CNF hydrogel (wt\%) & 1.0 & 1.5 & 2.0 & 2.5 \\
Density of CNF aerogel $\left(\mathrm{kg} / \mathrm{m}^{3}\right)$ & 11.2 & 16.2 & 19.9 & 26.7 \\
\hline
\end{tabular}




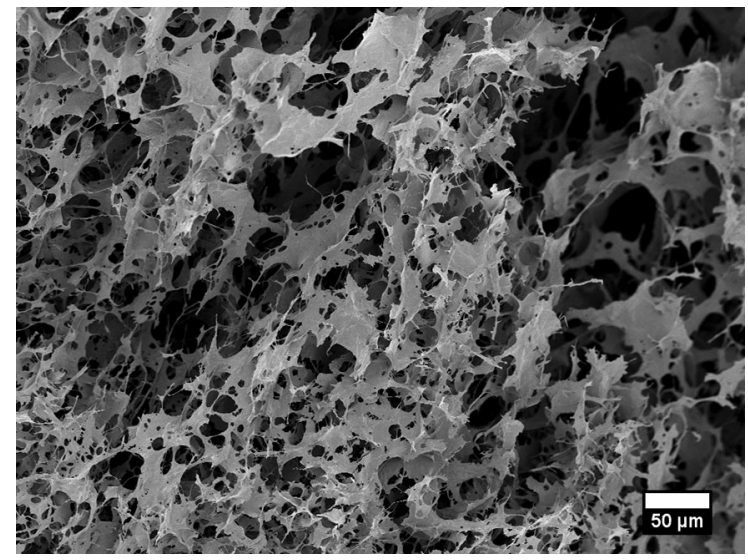

Fig. 7 SEM image of internal structure of the cross-linked CNF aerogel

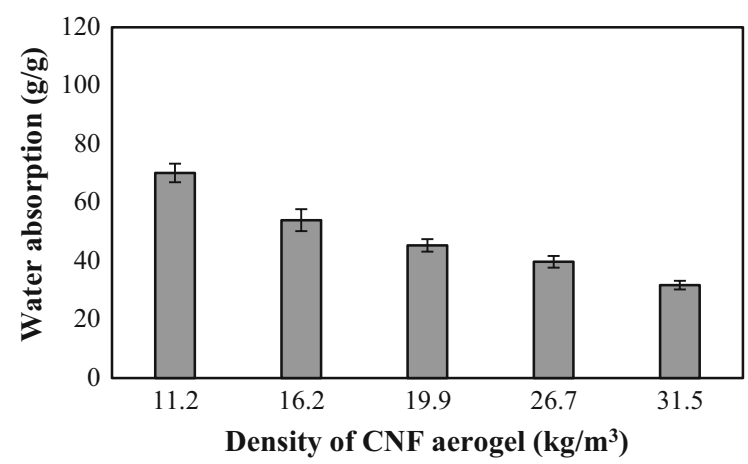

Fig. 8 Water absorbency of cross-linked CNF aerogel

capillary action due to the hydrophilic and porous nature of $\mathrm{CNF}$ aerogels. Because hydrogen bonding and mechanical entanglement of fibers are the main source of structural rigidity of the CNF aerogel, its network structure is destroyed and irreversibly collapsed upon the absorption of water. On the other hand, the cross-linked CNF aerogel retained its original shape and volume, and its water-absorbing capacity corresponded to its internal pore volume (Fig. 8).

Moisture uptake and its effect on the mechanical strength

As shown in Fig. 9, the equilibrium moisture content of cross-linked CNF aerogel was drastically increased with the relative humidity of air, and it was significantly greater for the typical handsheets prepared with

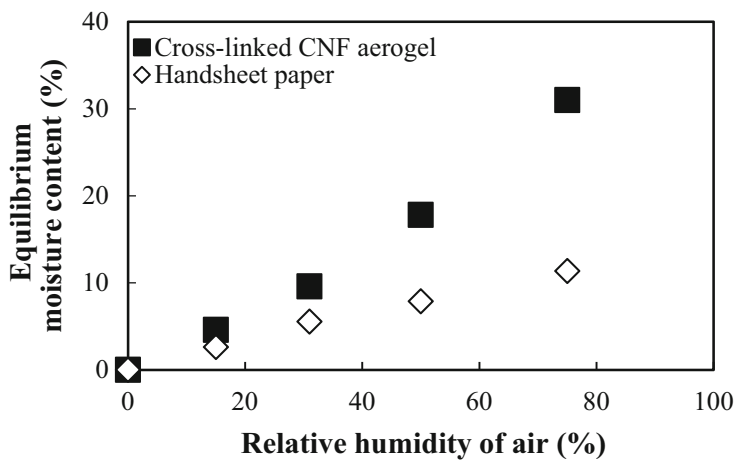

Fig. 9 Equilibrium moisture contents of cross-linked CNF aerogel and typical handsheet paper with a basis weight of $150 \mathrm{~g} / \mathrm{m}^{2}$ as a function of relative humidity of air

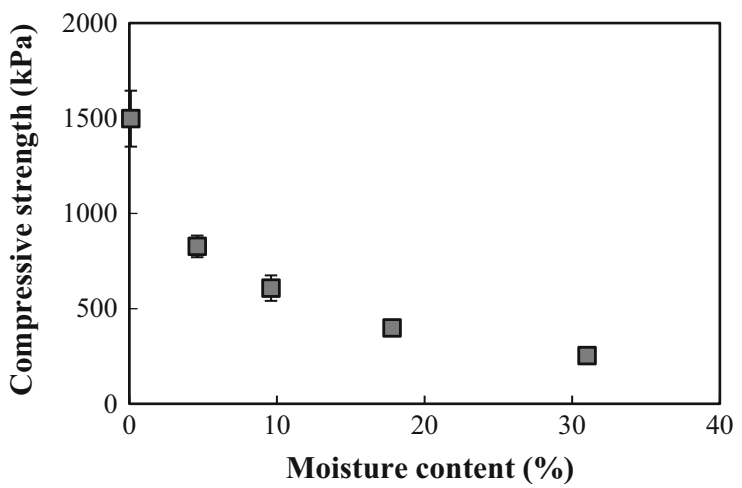

Fig. 10 Compressive strength of cross-linked CNF aerogel versus moisture content

the same bleached eucalyptus kraft pulp. At $75 \% \mathrm{RH}$ condition, the moisture contents of the cross-linked CNF aerogel was $31 \%$, while that of the handsheet was $11 \%$. The large specific surface area of the crosslinked CNF aerogel resulted in greater adsorption of water and gave higher equilibrium moisture content.

In Fig. 10, the compressive strength of cross-linked $\mathrm{CNF}$ aerogel was plotted as a function of the moisture content. The density of the cross-linked CNF aerogel was $31.5 \mathrm{~kg} / \mathrm{m}^{3}$. The compressive strength was drastically decreased as the moisture content was increased. The adsorption of water reduces the interfiber hydrogen bond of adjacent celluloses (Kang and Kim 2012; Kim et al. 2006). The reduction of compressive strength indicated that a considerable portion of the CNF network structure is still consisted of hydrogen bond even after the cross-linking treatment. 


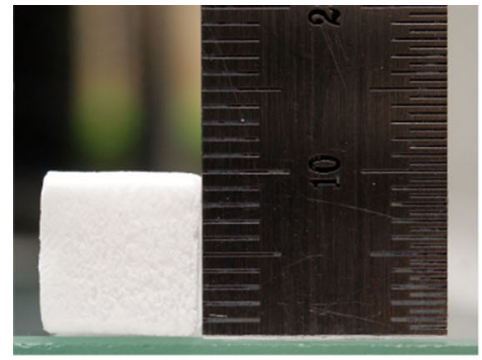

Dry state

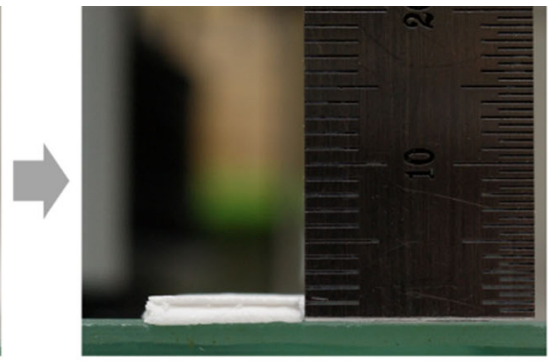

$90 \%$ compression

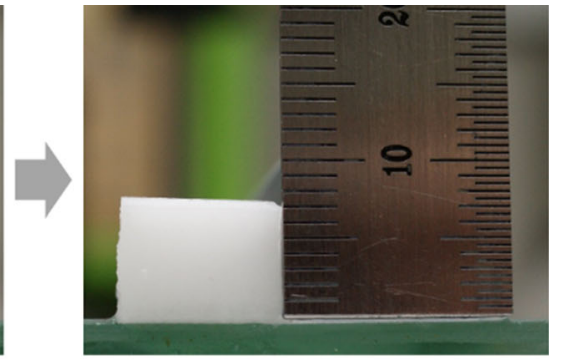

Wet state

Fig. 11 Shape recovery of cross-linked CNF aerogel by absorbing water

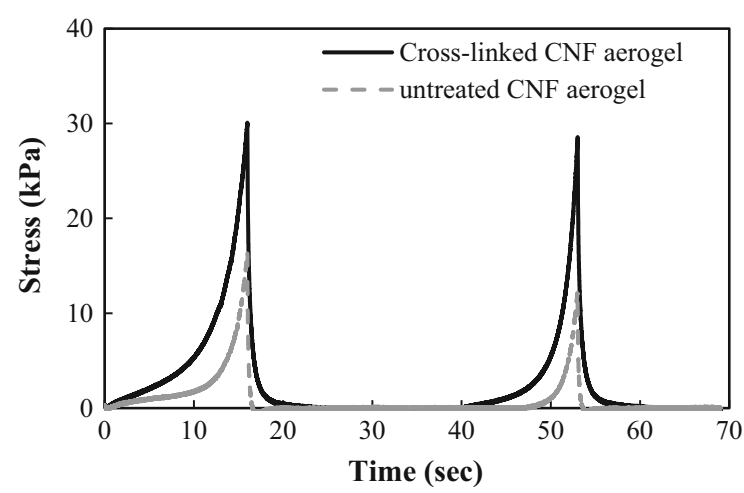

Fig. 12 Time-stress curve in TPA test of cross-linked and untreated $\mathrm{CNF}$ aerogel in wet state

Shape recovery of the cross-linked CNF aerogel

Cross-linking of CNF aerogel caused difference in the shape-recovery behavior after compressive deformation. Compressed CNF aerogels remained in compressed shape in dry state irrespective of the crosslinking treatment (Fig. 11). After wetting, the compressed cross-linked CNF aerogels showed substantially greater shape recovery characteristics. The cross-linked CNF aerogel recovered nearly $70 \%$ of the original shape within a few seconds after wetting. On the other hand, untreated CNF aerogel remained compressed even after wetting with water. The shape recovery property of cross-linked CNF aerogel in the wet state was examined with the use of TPA test (Fig. 12). The cross-linked CNF aerogel recovered its original volume after wet compression and the springiness value was 0.78 . On the other hand, the untreated CNF aerogel gave the springiness of 0.33 .
Zhang et al. (2012) described that the shaperecovery of the cross-linked CNF aerogels stems from the interaction between the absorbed water and CNF. Compression of the fiber network of CNF aerogel takes place by bending of individual fibers or breaking of interfiber bonds, and by the loss of both friction between adjacent fibers and physical entanglement of the fibers. Since the longitudinal compression of cellulose fibrils can be neglected, only the bending of the fiber can be taken into account during the compressive deformation of CNF aerogels. The elastic modulus of the crystal region of cellulose ranges from 130 to $250 \mathrm{GPa}$ (Zimmermann et al. 2004; Sakurada et al. 1962), while that of the amorphous region is reported to be 12.2 (Wang et al. 2014) or $10.4 \mathrm{GPa}$ (Chen et al. 2004). Due to this substantial difference in the elastic moduli in the crystalline and amorphous regions of cellulose, the bending of CNFs under compression is confined to the amorphous region. When the compressed crosslinked CNF aerogel absorb water, the amorphous region swells partially, causing the CNF to expand to a more stretched form and recovers its original shape. And the presence of connecting points in the crosslinked CNF aerogels caused the stretching of CNF transforms into structural expansion. In other words, the network structure of cross-linked CNF provides improved mechanical stability both in dry and wet states. As a result, the cross-linked CNF aerogel maintains its original interfiber network structure during the compression and recovery processes. When the external force is removed, the deformed individual fibers recover their original shape, and this expanding force allows the entire network return back closer to its original shape. 


\section{Conclusions}

Cross-linked CNF aerogels were prepared using maleic acid and sodium hypophosphite. The crosslinking reaction was composed of two steps; (1) grafting of maleic acid onto CNF via an esterification process, and (2) formation of cellulose cross-linking by a reaction between the grafted maleic acid and hypophosphite. The prepared cross-linked CNF aerogel showed improved network stability in a wet state indicating that the cross-linking reactions between $\mathrm{CNF}$ were obtained. The water absorbency of the cross-linked CNF decreased from 70.2 to $31.9 \mathrm{~g} / \mathrm{g}$ when the density of the cross-linked CNF aerogel was $11.2-31.5 \mathrm{~kg} / \mathrm{m}^{3}$. The moisture uptake of the crosslinked CNF aerogel at a given relative humidity increased significantly compared to a typical filter paper because of its high specific surface area. The cross-linked CNF aerogel showed typical plastic deformation behavior in dry state. When the crosslinked CNF aerogel absorbs water, it recovered its original shape. The cross-linked CNF aerogel showed higher springiness value than uncross-linked one.

Open Access This article is distributed under the terms of the Creative Commons Attribution 4.0 International License (http:// creativecommons.org/licenses/by/4.0/), which permits unrestricted use, distribution, and reproduction in any medium, provided you give appropriate credit to the original author(s) and the source, provide a link to the Creative Commons license, and indicate if changes were made.

\section{References}

Alemdar A, Sain M (2007) Isolation and characterization of nanofibers from agricultural residues-wheat straw and soy hulls. Bioresour Technol 99(6):1664-1671. doi:10. 1016/j.biortech.2007.04.029

Aulin C, Netrval J, Wågberg L, Lindström T (2010) Aerogels from nanofibrillated cellulose with tunable oleophobicity. Soft Matter 6(14):3298-3305. doi:10.1039/C001939A

Cai J, Liu S, Feng J, Kimura S, Wada M, Kuga S, Zhang L (2012) Cellulose-silica nanocomposite of TEMPO-oxidized MFC and CTMP fibres. Angew Chem Int Ed 51(9):2076-2079. doi:10.1002/anie.201105730

Cervin NT, Aulin C, Lasson PT, Wågberg L (2012) Ultra porous nanocellulose aerogels as separation medium for mixtures of oil/water liquids. Cellulose 19(2):401-410. doi:10.1007/ s10570-011-9629-5

Chen W, Lickfield GC, Yang CQ (2004) Molecular modeling of cellulose in amorphous state. Part I: model building and plastic deformation study. Polymer 45(3):1063-1071. doi:10.1016/j.polymer.2003.11.020
Chen W, Haipeng Y, Qing L, Yixing L, Jian L (2011) Ultralight and highly flexible aerogels with long cellulose I nanofibers. Soft Matter 7(21):10360-10368. doi:10.1039/ C1SM06179H

Dufresne A, Cavaillé JY, Helbert W (1997) Thermoplastic nanocomposites filled with wheat Straw cellulose whiskers. Part II: effect of processing and modeling. Polym Compos 18(2):198-210. doi:10.1002/pc.10274

Heiskanen L, Backfolk K, Vehviläinen M, Kamppuri T, Nousiainen P (2012) Process for producing microfibrillated cellulose. Eur Pat 2011004301:28

Henriksson M, Henriksson G, Berglund LA, Lidström T (2007) An environmentally friendly method for enzyme-assisted preparation of microfibrillated cellulose (MFC) fibers. Eur Polym J 43(8):3434-3441. doi:10.1016/j.eurpolymj. 2007. 05.038

Herrick FW, Casebier RL, Hamilton JK, Sandberg KR (1983) Microfibrillated cellulose: morphology and accessibility. J Appl Polym Sci: Appl Polym Symp 37:797-813

Hüsing N, Schubert U (1998) Aerogels-airy materials: chemistry, structure, and properties. Angew Chem Int Ed 37(1-2):22-45. doi:10.1002/(SICI)1521-3773(19980202) $37: 1 / 2<22$ :AID-ANIE22>3.0.CO;2-I

Isogai T, Saito T, Isogai A (2011) Wood cellulose nanofibrils prepared by TEMPO electro-mediated oxidation. Cellulose 18(2):421-431. doi:10.1007/s10570-010-9484-9

Iwamoto S, Nakagaito AN, Yano H, Nogi M (2005) Optically transparent composites reinforced with plant fiber-based nanofibers. Appl Phys A 81(6):1109-1112. doi:10.1007/ s00339-005-3316-z

Iwamoto S, Nakagaito AN, Yano H (2007) Nano-fibrillation of pulp fibers for the processing of transparent nanocomposites. Appl Phys A 89(2):461-466. doi:10.1007/s00339007-4175-6

Kang K, Kim H (2012) Effects of temperature and relative humidity on the physical properties of electronic copying paper. J Korea TAPPI 44(3):70-78

Kim H, Choi W, Um G (2006) The effect of atmospheric conditions on the physical and mechanical properties of linerboard. J Korea TAPPI 38(5):60-65

Kistler SS (1931) Coherent expanded aerogels and jellies. Nature 127:741. doi:10.1038/127741a0

Missoum K, Belgacem MN, Bras J (2013) Nanofibrillated cellulose surface modification: a review. Materials 6(5): 1745-1766. doi:10.3390/ma6051745

Nakagaito AN, Yano H (2004) The effect of morphological changes from pulp fiber towards nano-scale fibrillated cellulose on the mechanical properties of high-strength plant fiber based composites. Appl Phys A 78(4):547-552. doi:10.1007/s00339-003-2453-5

Pääkkö M, Ankerfors M, Kosonen H, Nykänen A, Ahola S, Österberg M (2007) Enzymatic hydrolysis combined with mechanical shearing and high-pressure homogenization for nanoscale cellulose fibrils and strong gels. Biomacromolecules 8(6):1934-1941. doi:10.1021/bm061215p

Pääkkö M, Vapaavuori J, Silvennoinen R, Kosonen H, Ankerfors M, Lindström T, Berglund LA, Ikkala O (2008) Long and entangled native cellulose I nanofibers allow flexible aerogels and hierarchically porous templates for functionalities. Soft Matter 4:2492-2499. doi:10.1039/B810371B 
Pierre AC, Pajonk GM (2002) Chemistry of aerogels and their applications. Chem Rev 102(11):4243-4266. doi:10.1021/ cr0101306

Saito T, Nishiyama Y, Putaux J, Vignon M, Isogai A (2006) Homogeneous suspensions of individualized microfibrils from TEMPO-catalyzed oxidation of native cellulose. Biomacromolecules 7(6):1687-1691. doi:10.1021/bm06 $0154 \mathrm{~s}$

Saito T, Kimura S, Nishiyama Y, Isogai A (2007) Cellulose nanofibers prepared by TEMPO-mediated oxidation of native cellulose. Biomacromolecules 8(8):2485-2491. doi:10.1021/bm0703970

Sakurada I, Nukushina Y, Ito T (1962) Experimental determination of the elastic modulus of crystalline regions in oriented polymers. J Polym Sci 57(165):651-660. doi:10. 1002/pol.1962.1205716551

Sehaqui H, Salajková M, Zhou Q, Berglund LA (2010) Mechanical performance tailoring of tough ultra-high porosity foams prepared from cellulose I nanofiber suspensions. Soft Matter 6(8):1824-1832. doi:10.1039/ B927505C

Shibata M, Nakai M (2010) Preparation and properties of biocomposites composed of bio-based epoxy resin, tannic acid, and microfibrillated cellulose. J Polym Sci B 48(4):425-433. doi:10.1002/polb.21903

Sim K, Ryu J, Youn HY (2015) Structural characteristics of nanofibrillated cellulose mats: effect of preparation conditions. Fibers Polym 16(2):294-301. doi:10.1007/s12221015-0294-4

Siqueira G, Bras J, Dufresne A (2010) Luffa cylindrica as a lignocellulosic source of fiber, microfibrillated cellulose, and cellulose nanocrystals. BioResources 5(2):727-740

Svagan AJ, Azizi Samir MAS, Berglund LA (2008) Biomimetic foams of high mechanical performance based on nanostructured cell walls reinforced by native cellulose nanofibrils. Adv Mater 20(7):1263-1269. doi:10.1002/ adma.200701215

Syverud K, Kirsebom H, Hajizadeh S, Chinga-Carrasco G (2011) Cross-linking cellulose nanofibrils for potential elastic cryo-structured gels. Nanoscale Res Lett 6:626-632. doi:10.1186/1556-276X-6-626

Syverud K, Pettersen SR, Draget K, Chinga-Carrasco G (2015) Controlling the elastic modulus of cellulose nanofibril hydrogels-scaffolds with potential in tissue engineering. Cellulose 22(1):473-481. doi:10.1007/s10570-014-0470-5
Taipale T, Österberg M, Nykänen A, Ruokolainen J, Lainen J (2010) Effect of microfibrillated cellulose and fines on the drainage of kraft pulp suspension and paper strength. Cellulose 17(5):1005-1020. doi:10.1007/s10570-0109431-9

Turbak AF, Synder FW, Sandberg KR (1983) Microfibrillated cellulose, a new cellulose product: properties, uses, and commercial potential. J Appl Polym Sci: Appl Polym Symp 37:815-827

Uetani K, Yano H (2011) Nanofibrillation of wood pulp using a high-speed blender. Biomacromolecules 12(2):348-353. doi:10.1021/bm101103p

Wang YY, Tian M, Xu HX, Fan P (2014) Influence of moisture on mechanical properties of cellulose insulation paper. Int $\mathrm{J}$ Mod Phys B 28(7):1450051-1-1450051-12. doi: 10.1142/ S0217979214500519

Yang CQ, Chen D, Guan J, He Q (2010) Cross-linking cotton cellulose by the combination of maleic acid and sodium hypophosphite. 1. Fabric wrinkle resistance. Ind Eng Chem Res 49(18):8325-8332. doi:10.1021/ie1007294

Yano H, Nakahara S (2004) Bio-composites produced from plant microfiber bundles with a nanometer unit web-like network. J Mater Sci 39(5):1635-1638. doi:10.1023/B: JMSC.0000016162.43897.0a

Yao W, Wang B, Ye T, Yang Y (2013) Durable finishing of cotton fabrics with citric acid: enhancement of whiteness and wrinkle recovery by polyol extenders. Ind Eng Chem Res 52(46):16118-16127. doi:10.1021/ie402747x

Zhang W, Yaan Z, Canhui L, Yulin D (2012) Aerogels from crosslinked cellulose nano/micro-fibrils and their fast shape recovery property in water. J Mater Chem 22(3):11642-11650. doi:10.1039/C2JM30688C

Zheng Q, Cai Z, Gong S (2014) Green synthesis of polyvinyl alcohol (PVA)-cellulose nanofibril (CNF) hybrid aerogels and their use as superabsorbents. J Mater Chem A 2(9):3110-3118. doi:10.1039/C3TA14642A

Zimmermann T, Pohler E, Geiger T (2004) Cellulose fibrils for polymer reinforcement. Adv Eng Mater 6(9):754-761. doi:10.1002/adem.200400097

Zimmermann T, Bordeanu N, Strub E (2010) Properties of nanofibrillated cellulose from different raw materials and its reinforcement potential. Carbohydr Polym 79(4): 1086-1093. doi:10.1016/j.carbpol.2009.10.045 